Clinical Vistas

\section{A tourist with tungiasis}

A 35-year-old man presented with a slightly painful papule with a central dark spot on his right heel (Figure I). It had developed 2 weeks before his return to Canada after a 3-month vacation in Ecuador. The lesion was typical of tungiasis, which is endemic to Ecuador. A skin biopsy revealed the characteristic exoskeleton of Tunga penetrans, as well as its internal organs and eggs just beneath the stratum corneum (Figure 2). Excision of the lesion under aseptic conditions was performed (Figure 3).

Tungiasis is caused by an infestation of gravid female $T$. penetrans. $T$. penetrans are also referred to as chigoe, jigger and sand flea. Common hosts include humans, dogs, cats and pigs. This ectoparasite is usually acquired by walking barefoot in the sandy soil of disease-endemic regions, including the Caribbean, Latin America, Africa and India. Although the fleas are only about I mm long, they can jump up to $20 \mathrm{~cm}$ vertically and can crawl or run up to I cm per second. ${ }^{1}$ Both male and female fleas are hematophagous. ${ }^{2}$ The male does not burrow into the host and dies after copulation. However, the female flea burrows into the host's epidermis, where it can grow as large as I $\mathrm{cm}$ as its fertilized eggs mature. ${ }^{2}$ Penetration of the skin is usually complete within 3 hours and manifests clinically as a black dot, corresponding to the anal-genital opening through which the flea breathes and expels feces and eggs. ${ }^{2}$ Females lay I50-200 eggs over a period of $2-3$ weeks and then die. Most of the eggs will fall to the ground where the larvae hatch within 3-4 days. The larvae develop into pupae IO-I5 days later, and the pupae reach adulthood after an additional 5-I4 days. Under optimum conditions, the transition from egg to adult can be as brief as 18 days.

Tungiasis is characterized by a papular or nodular lesion with a central dark spot. It most commonly affects the feet,

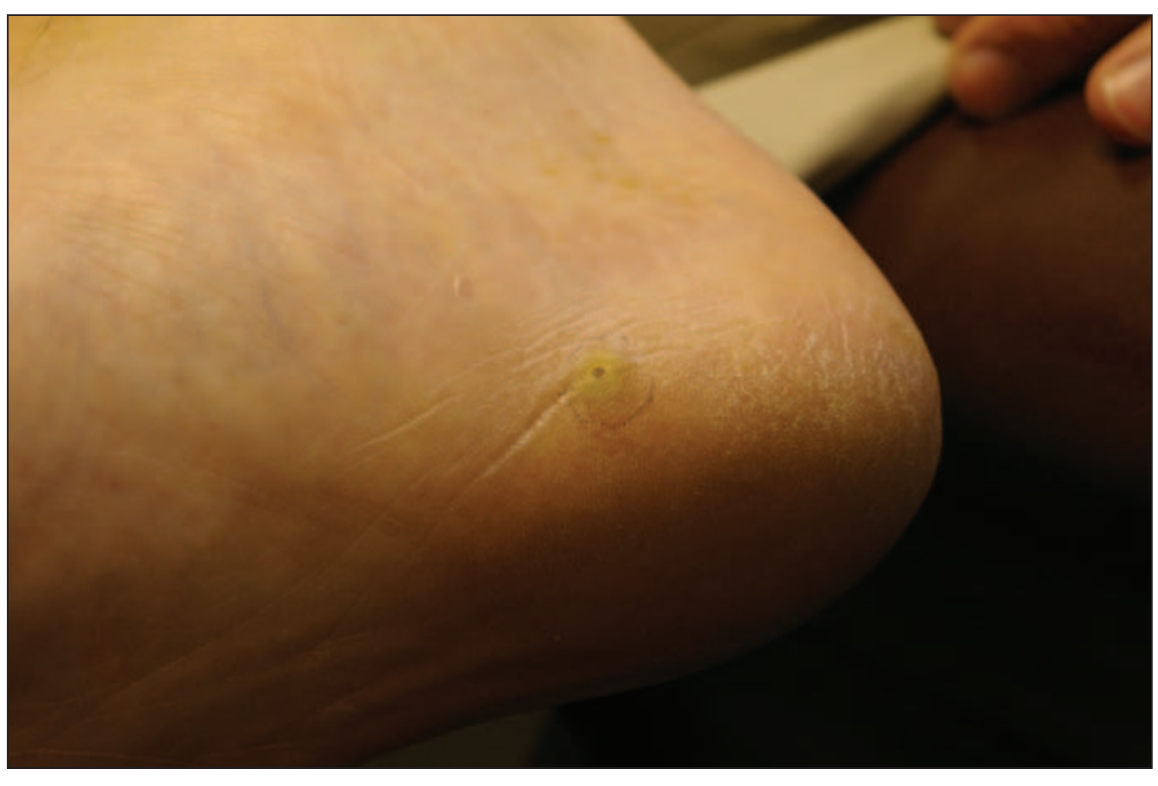

Figure 1: Papule with a small dark spot in the patient's right heel.

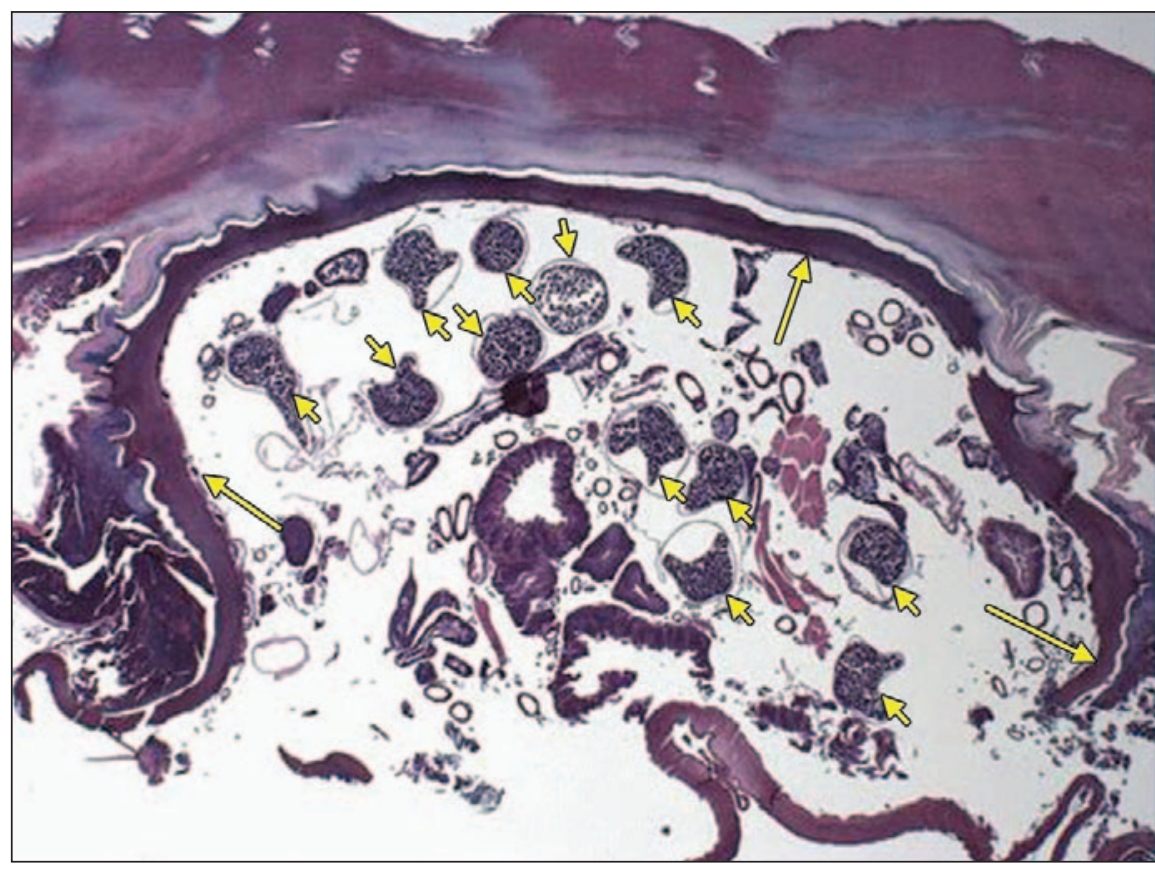

Figure 2: Histopathologic image of the lesion on the patient's heel. Note the characteristic exoskeleton of Tunga penetrans (long arrows) and the internal organs and eggs (short arrows) beneath the stratum corneum (hematoxylin-eosin stain, original magnification $\times 25$ ).

although the involved region depends on the point of contact with the sand. Lesions can be pruritic, painful or asymptomatic. The differential diagnosis includes myiasis, cutaneous larva migrans, dracunculosis, scabies, tick bite, verruca and foreign body. Complications include cellulitis, lymphangitis, ulceration, septicemia, gangrene and tetanus. Involvement of the nail matrix can lead to nail deformation or loss.

Definitive treatment consists of sterile curettage or excision. Topical ivermectin, metrifonate or thiabendazole therapy has been used with success to reduce the number of lesions caused by the embedded fleas. ${ }^{3}$ Many dermatologists claim that ivermectin is effective 
based on their personal experience and published case reports; ${ }^{4}$ however, controlled trials have not been performed. Topical antibiotic therapy is used to prevent bacterial infection, and oral antibiotic therapy is indicated for the management of secondary infection. The patient's tetanus vaccination status should be confirmed in all cases.
Tungiasis is highly prevalent in many resource-poor communities in developing countries, where the prevalence may exceed 50\%. Since travel to these countries is popular, physicians should be familiar with the clinical manifestations of tungiasis and the appropriate treatment of this exotic infestation. Preventive measures for

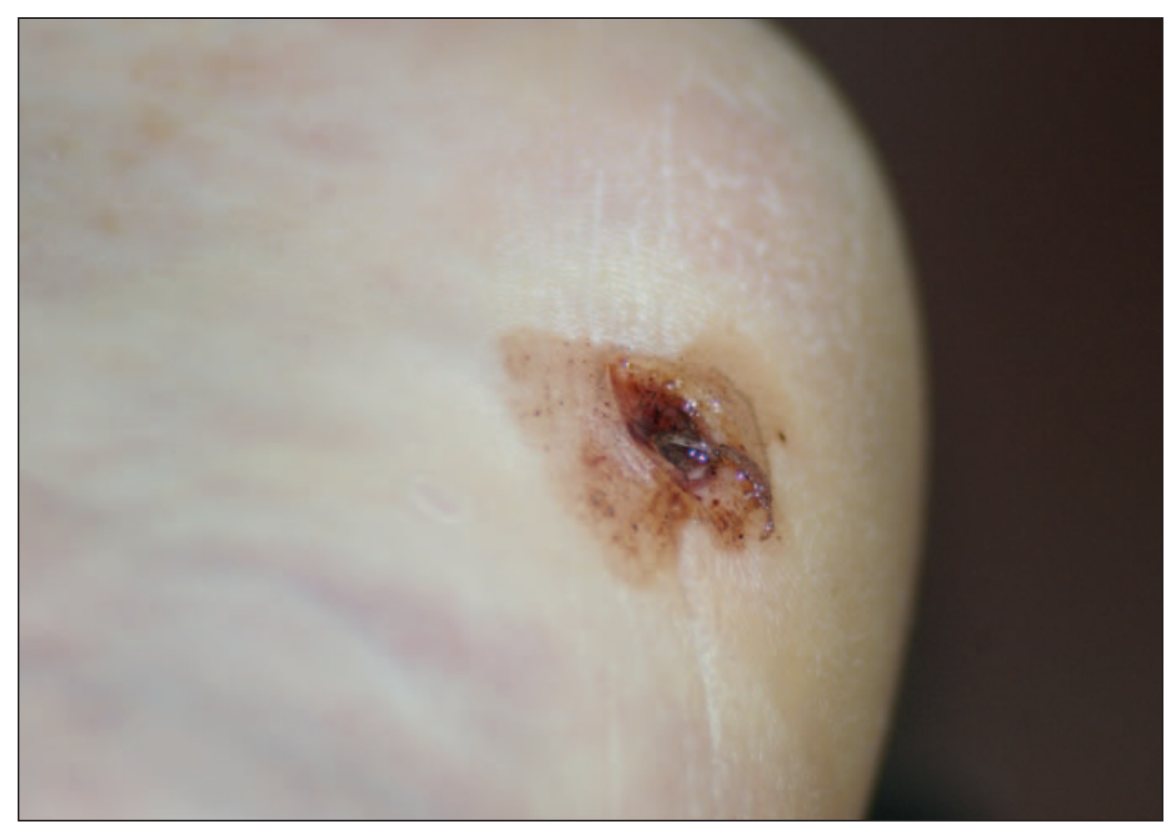

Figure 3: Excision of the lesion was performed under aseptic conditions. those who travel to tungiasis-endemic areas include the use of footwear and insect repellent.

\section{Alexander K.C. Leung MBBS \\ Department of Pediatrics \\ University of Calgary \\ Tom Woo MD \\ Department of Medicine \\ University of Calgary \\ William Lane M. Robson MD \\ The Childrens' Clinic \\ Calgary, Alta. \\ Martin J. Trotter MD \\ Department of Pathology and \\ Laboratory Medicine \\ University of Calgary \\ Calgary, Alta.}

\section{This article has been peer reviewed.}

Competing interests: None declared.

\section{REFERENCES}

I. Eisele M, Heukelbach J, Van Marck E, et al. Investigations on the biology, epidemiology, pathology and control of Tunga penetrans in Brazil: I. Natural history of tungiasis in man. Parasitol Res 2003;90:87-99.

2. Fein $\mathrm{H}$, Naseem $\mathrm{S}$, Witte $\mathrm{D}$, et al. Tungiasis in North America: a report of 2 cases in internationally adopted children. J Pediatr 200I;139:744-6.

3. Heukelbach J, Eisele M, Jackson A, et al. Topical treatment of tungiasis: a randomized, controlled trial. Ann Trop Med Parasitol 2003;97:743-9.

4. Heukelbach J. Tungiasis. Rev Inst Med Trop Sao Paulo 2005;47:307-I3.

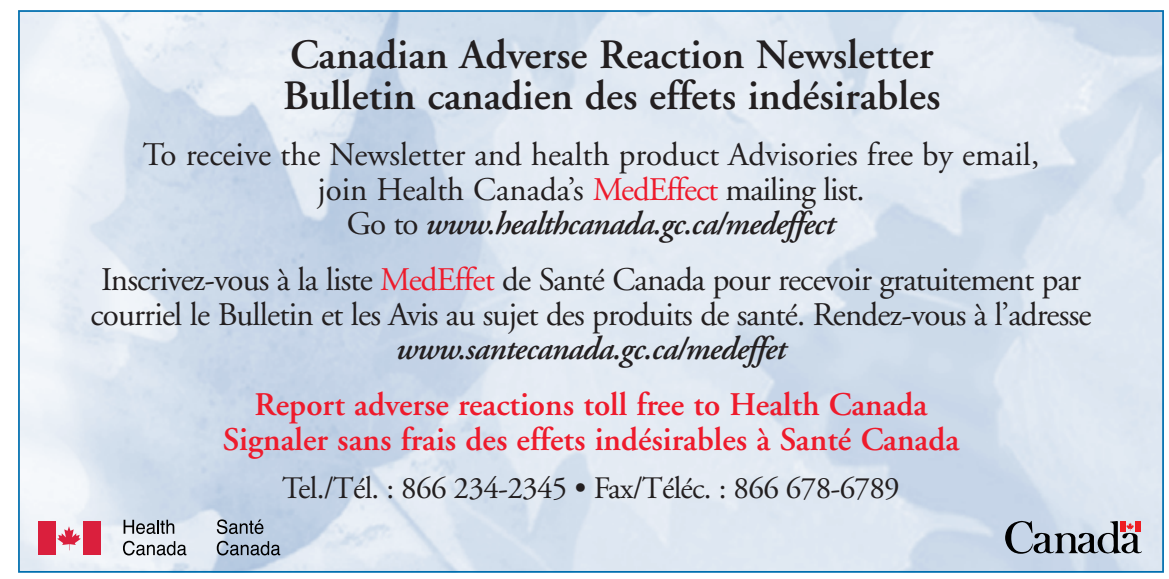

